\title{
First-time anterior shoulder dislocations: should they be arthroscopically stabilised?
}

\author{
Sedeek Mohamed Sedeek ${ }^{1}$, MCH, FRCSI, Hamid Rahmatullah Bin Abd Razak ${ }^{2}$, MBBS, MRCS,
} Gerard WW $\underline{E e}^{2}$, MBBS, MRCS, Andrew $\mathrm{HC} \underline{\operatorname{Tan}^{2}}$, FRCSG, FRCSE

\begin{abstract}
The glenohumeral joint is inherently unstable because the large humeral head articulates with the small shadow glenoid fossa. Traumatic anterior dislocation of the shoulder is a relatively common athletic injury, and the high frequency of recurrent instability in young athletes after shoulder dislocation is discouraging to both the patient and the treating physician. Management of primary traumatic shoulder dislocation remains controversial. Traditionally, treatment involves initial immobilisation for 4-6 weeks, followed by functional rehabilitation. However, in view of the high recurrence rates associated with this traditional approach, there has been an escalating interest in determining whether immediate surgical intervention can lower the rate of recurrent shoulder dislocation, improving the patient's quality of life. This review article aims to provide an overview of the nature and pathogenesis of first-time primary anterior shoulder dislocations, the widely accepted management modalities, and the efficacy of primary surgical intervention in first-time primary anterior shoulder dislocations.
\end{abstract}

Keywords: arthroscopic Bankart repair, conservative treatment, dislocation, shoulder

\section{INTRODUCTION}

The glenohumeral joint is inherently unstable because the large humeral head articulates with the small shadow glenoid fossa, and it is the most frequently dislocated joint. ${ }^{(1)}$ The stability of the glenohumeral joint is maintained by the glenoid labrum. This labrum creates a socket-deepening effect, hence preventing shoulder dislocation. The anteroinferior labrum also serves as the anchor point for the inferior glenohumeral ligament, the primary static restraint to the anterior humeral translation in the abducted shoulder. ${ }^{(2,3)}$ An avulsion of the labrum from the glenoid rim is known as a Bankart lesion. ${ }^{(3)}$

Traumatic anterior dislocation of the shoulder is a relatively common injury in the young and active population. The high frequency of recurrent instability in young athletes after shoulder dislocation is discouraging to both the patient and the treating physician..$^{(4,5)}$ Many factors, including age, athletic participation, length of immobilisation and length of rehabilitation, have been evaluated to determine their effects on the recurrence of instability. ${ }^{(4)}$

The management of primary traumatic shoulder dislocation remains controversial. Traditionally, treatment consists of initial immobilisation, ranging from four to six weeks, followed by intensive functional rehabilitation. ${ }^{(5)}$ However, in view of the high recurrence rates associated with this approach, there has been an interest in determining whether immediate surgical intervention is able to lower the rate of recurrent shoulder dislocation and to improve the patient's quality of life. ${ }^{(6)}$ This review article aims to provide an overview of: (a) the nature and pathogenesis of first-time primary anterior shoulder dislocations; (b) the widely accepted management modalities; and (c) the efficacy of primary surgical intervention for this condition.

\section{PATHOLOGY OF ACUTE DISLOCATION}

The shallow glenohumeral joint sacrifices its stability for an extensive range of motion. Joint restraints are normally categorised into two types: (a) static stabilisers, such as the glenoid fossa, labrum, joint capsule and glenohumeral ligaments; and (b) dynamic stabilisers, such as the rotator cuff, long head of biceps and stabilisers of the scapula. ${ }^{(7)}$ Anterior shoulder dislocations usually occur with the arm in an abducted and externally rotated position, leading to predictable patterns of injury to the labrum, capsuloligamentous structures, glenoid and humeral head. During traumatic anterior dislocation, the humeral head is displaced in an anteroinferior manner; this stretches the capsuloligamentous components, often resulting in a Bankart lesion, which has been regarded as the essential lesion in such cases. ${ }^{(7)}$ Other lesions that may be associated with traumatic anterior dislocations include bony Hill-Sachs lesions, superior labral tears from anterior to posterior (SLAP), capsular tears, rotator cuff tears and glenoid rim fractures. ${ }^{(8)}$

In a study evaluating first-time traumatic dislocations in young patients, Taylor and Arciero ${ }^{(9)}$ documented that $97 \%$ of their patients had Bankart lesions with no gross evidence of capsular injury. They also noted that $89 \%$ of their patients had Hill-Sachs lesions, although the lesions were small and did not appear to significantly affect stability on arthroscopic evaluation. ${ }^{(9)}$ Similar findings were observed by Baker et al's study, ${ }^{(10)}$ which reported an $87 \%$ incidence of Bankart lesions, a $64 \%$ incidence of Hill-Sachs bony injury, and an $18 \%$ incidence of capsular tear cum rotator cuff injuries. ${ }^{(10)}$ Antonio et al, who evaluated abnormalities in first-time dislocations using magnetic resonance (MR) arthrography, found that $82 \%$ of patients aged under 30 years had an anteroinferior labral avulsion, $71 \%$ had Hill-Sachs lesions and $14 \%$ had SLAP lesions. ${ }^{(11)}$

${ }^{1}$ Department of Orthopaedic Surgery, East Lancashire Hospitals, Royal Blackburn Hospital, Blackburn, UK, ²Department of Orthopaedic Surgery, Singapore General Hospital, Singapore

Correspondence: Dr Sedeek Mohamed Sedeek, Department of Orthopaedic Surgery, East Lancashire Hospitals, Royal Blackburn Hospital, Haslingden Road, Blackburn, Lancashire, BB2 3HH, UK. sedeeko2000@hotmail.com 
For the purpose of discussing the rationale of various treatments for first-time dislocation, some authors have studied the drawbacks of additional recurrent instability events. For instance, Pollock et $\mathrm{al}^{(12)}$ studied the mechanical response of the inferior glenohumeral ligament to varying sub-failure cyclic strain in 33 fresh frozen human cadaveric shoulders, their results demonstrating that repetitive loading of the inferior glenohumeral ligament induced laxity and irreversible elongation in the ligament, as manifested in the peak load response and measured elongations. They also noted that mechanical response of the ligament is affected by both the magnitude of the cyclic strain and the frequency of loading at higher strain levels. ${ }^{(12)}$

A study by Buscayret et $\mathrm{al}^{(13)}$ which examined the bony lesions of 570 patients who underwent a stabilising procedure, found that the development of postoperative arthritis correlated with a higher number of preoperative dislocations. Furthermore, they identified that the presence of osseous glenoid rim lesions was a risk factor in the development of preoperative arthritis. ${ }^{(13)}$ Griffith et al ${ }^{(14}$ assessed the relationship between the frequency of dislocation and the prevalence, pattern and spectrum of glenoid bone loss in anterior shoulder dislocation, utilising computed tomography (CT). They found that glenoid bone loss was present in $41 \%$ of their patients with first-time dislocation and $86 \%$ of their patients with recurrent dislocation, with the number of dislocations moderately correlating with the severity of glenoid bone loss. ${ }^{(14}$

\section{TREATMENT OF INITIAL DISLOCATION Clinical assessment and primary treatment}

Detailed history-taking and examination are important in the assessment of patients with primary anterior shoulder dislocation. ${ }^{(7)}$ Careful documentation of any neurovascular deficit, especially axillary nerve palsy, is mandatory. Various types of brachial plexus lesions and isolated nerve palsies have also been described in association with traumatic anterior dislocations of the shoulder. ${ }^{(7)}$

Anteroposterior and modified axillary radiographies are crucial for confirming the dislocation and the presence of any associated fractures, before any attempt at manipulation and reduction is made. Post-reduction radiography is also mandatory to confirm a congruent reduction, as well as to reassess the position of any associated fractures. ${ }^{(7,15)}$ While CT is an option when complex bony damage is suspected, MR imaging is now the gold standard for assessing soft tissue injury in cases with anterior instability. MR arthrography is superior to other imaging methods in revealing ligament or capsular detachments, tears of the rotator cuff, damage to the articular cartilage, and labral lesions. ${ }^{(16)}$

\section{Conservative management}

Reduction should be done carefully to avoid any additional bony or soft tissue injury. Kuhn reported that patients who were premedicated with intra-articular lidocaine had fewer complications and required a shorter time in the emergency department, as compared to patients who were premedicated with intravenous sedation, which usually requires a longer observation time in the emergency department. ${ }^{(17)}$ Notably, there was no significant difference in the success of reduction of the glenohumeral joint with either type of premedication. ${ }^{(17)}$

Some studies assessed the length and position of post-reduction immobilisation. Hovelius et al found no significant difference in the recurrence rates of patients who were immobilised in internal rotation for one week vs. 3-4 weeks. ${ }^{(18)}$ Likewise, Simonet and Cofield reported that the duration of immobilisation had no influence on dislocation recurrence rate; the only treatment factor that was found to influence the dislocation recurrence rate was related to restriction from sports and full activities for more than six weeks. ${ }^{(19)}$ Kiviluoto et al's study, which included patients aged less than 30 years with primary shoulder dislocations, found that the recurrence rate was $50 \%$ in the 26 patients who were immobilised for one week, while it was $22 \%$ in the 27 patients who were immobilised for three weeks. ${ }^{(20)}$

With regard to the position in which the affected arm should be immobilised, the traditional 'safe position', i.e. the shoulder held in internal rotation (IR), neutral flexion and abduction, with the elbow flexed at $90^{\circ}$, is often utilised. Although there are few comparative studies that evaluated the superiority of this position, some recent studies have shown that immobilisation with the arm in external rotation (ER) may reduce the risk of subsequent dislocation, as this position approximates the Bankart lesion to the neck of the glenoid, allowing better healing. ${ }^{(7)}$

Seybold et al performed standardised MR imaging of the shoulders in IR and ER positions after initial reduction in 34 patients with first-time traumatic anterior shoulder dislocation, and they found that, in the ER position, there was significant improvement in the position of the labroligamentous lesion on the glenoid rim. ${ }^{(21)}$ Itoi et al's study, ${ }^{(22)}$ which involved a total of 198 patients immobilised for three weeks in either IR or $10^{\circ}$ of $E R$, found a significantly lower recurrence rate in the ER group as compared to the IR group ( $26 \%$ vs. $42 \%$ ). They also noted that immobilisation in the ER position was particularly beneficial for patients aged 30 years or younger. ${ }^{(22)}$

Traditionally, recurrent instability has been used as the sole outcome measure of treatment. However, other considerations (e.g. continued apprehension, failure to return to work or sport, quality of life and development of posttraumatic osteoarthritis) are also crucial in assessing the outcomes of treatment. ${ }^{(8)}$ Many authors have studied the factors associated with recurrent instability. Age at the time of initial dislocation has been found to be the most important factor for recurrence of instability. ${ }^{(8)}$ Gumina and Postacchini reported a recurrence rate of $22 \%$ (mean follow-up period of seven years) in 545 consecutive patients with anterior shoulder dislocations who were conservatively treated. ${ }^{(23)}$ Hovelius et al's study, which examined the results of conservative treatment in primary anterior shoulder dislocations over a followup period of 25 years, found a recurrence rate of $72 \%$ in patients aged $12-22$ years, 56\% in patients aged $23-29$ years and $27 \%$ in patients older than 30 years. ${ }^{(24)}$

Sachs et al ${ }^{(25)}$ aimed to identify patients at high risk for shoulder redislocation and determine whether these high-risk patients were best served by immediate surgical stabilisation by prospectively 
following up on 131 patients for five years. They found that younger patients who were involved in contact or collision sports, or who required overhead occupational use of the arm, were more likely to have re-dislocation of the shoulder than their less active peers, or older persons. However, even in the highest-risk group, only approximately half of the patients with shoulder re-dislocation requested for surgical treatment within the follow-up period. Thus, the authors could not justify early surgery based on the presumption of future dislocations, unhappiness and disability. ${ }^{(25)}$

\section{Surgical treatment}

Many studies have evaluated surgical procedures that can be undertaken after an acute traumatic shoulder dislocation. A study by Wintzell et al, which aimed to evaluate the effect of arthroscopic lavage as a form of treatment for acute anterior glenohumeral dislocation, reported a recurrence rate of $43 \%$ in patients who received nonsurgical treatment, while that of patients who received arthroscopic lavage was only $13 \% .^{\text {(26) }}$

Robinson et $\mathrm{al}^{(27)}$ found that arthroscopic repair of a Bankart lesion after primary traumatic anterior shoulder dislocation reduced the risk of recurrence by $76 \%$; they also found that the risk of all recurrent instability was reduced by $82 \%$ in this group of patients that underwent Bankart repair, as compared to the group that underwent arthroscopy and lavage alone. They concluded that marked treatment benefit could be derived from primary arthroscopic repair of a Bankart lesion, distinct from the so-called background therapeutic effect of arthroscopic examination and lavage of the joint. However, they noted that primary repair did not appear to confer a functional benefit to patients with a stable shoulder at two years after the dislocation. ${ }^{(27)}$

In a study that evaluated the results of early arthroscopic Bankart repair in patients with primary traumatic anterior dislocation of the shoulder, Valentin et al $^{(28)}$ found that $93 \%$ of their patients had good or excellent results at 18 months. In addition, 83\% of their patients returned to their pre-dislocation activity level. In this study, the patients' age ranged from 17 to 34 years, and arthroscopic Bankart repair was performed within 12 days after dislocation. ${ }^{(28)}$ Similar results were obtained by Law et al in their study involving 38 young patients with first-time dislocation who were treated with initial arthroscopic Bankart repair using suture anchors; the re-dislocation rate after a minimum of two years of follow-up was $5.2 \%$. $^{(29)}$

\section{Surgical versus nonsurgical treatment}

There have been many studies conducted to address and advocate for optimal treatment of acute anterior shoulder dislocations. Bottoni et al, ${ }^{(30)}$ for instance, compared the outcomes of nonsurgical treatments with arthroscopic Bankart repair for acute, traumatic shoulder dislocations in two groups of young athletes. In their study, which had an average follow-up period of 36 months, the recurrence rate was $75 \%$ in the nonsurgical treatment group and $11.1 \%$ in the surgically treated patients. The authors concluded that early arthroscopic stabilisation is more predictable, and has lower recurrence rates and better outcomes in young athletic patients. ${ }^{(30)}$
Kirkley et $\mathrm{al}^{(31)}$ reported the long-term results of a prospective randomised clinical trial that aimed to compare the effectiveness of immediate arthroscopic stabilisation with that of immobilisation and rehabilitation after a first traumatic anterior dislocation of the shoulder. At an average follow-up period of 75 months, the authors found a significant difference in the rate of re-dislocation between the two treatment groups, and thus concluded that immediate arthroscopic stabilisation is the treatment of choice in patients younger than 30 years old and in higher-level athletes. ${ }^{(31)}$

The long-term results of surgical arthroscopic stabilisation and conservative primary treatment for first-time traumatic anterior shoulder dislocation were also compared in a study by Jakobsen et al. ${ }^{(32)}$ At follow-up two years after treatment, it was found that the recurrent instability rates were $54 \%$ for the nonsurgical treatment group and $10 \%$ for the surgically treated group. At follow-up ten years after treatment, the instability rates were $26 \%$ and $9 \%$, respectively. ${ }^{(32)}$ Yanmis et al ${ }^{(33)}$ compared the stability and functional outcomes of patients who underwent arthroscopic repair with an absorbable tack following the first traumatic dislocation with patients who underwent conservative treatment. In the latter, the average pre- and post-treatment Constant scores were 46.8 and 71.4 , respectively, whereas the surgically treated group had an average preoperative Constant score of 44.7 (in cases with Bankart lesion) and an average postoperative Constant score of 92.3. These results, therefore, support the use of absorbable tacks in arthroscopic repair of traumatic acute anterior shoulder dislocations. ${ }^{(33)}$

\section{WHAT OUTCOMES DO WE AIM TO ACHIEVE? \\ Proprioception}

In a randomised clinical trial comparing the effect of early arthroscopic stabilisation with that of standard immobilisation on measures of shoulder proprioception, Edmonds et al concluded that, after primary traumatic anterior dislocation of the shoulder, early treatment with arthroscopic stabilisation does not enhance proprioception more than standard immobilisation and rehabilitation. ${ }^{(34)}$

\section{Quality of life}

In 1999, Kirkley et al ${ }^{(35)}$ reported statistically significant improvements in the quality of life of patients treated with arthroscopic stabilisation as compared to those who were treated nonsurgically. Using the Western Ontario Shoulder Instability index for assessment, they observed improvements in four components, namely physical symptoms, sport function, lifestyle and social function, and emotional wellbeing. ${ }^{(35)}$

\section{ADVOCATING SURGERY}

The three major reasons cited in the literature for supporting immediate stabilisation over conservative treatment are: (a) the unacceptable high risk of recurrence in the young athletic population; (b) the recurrent instability that propagates significant and progressive soft tissue and bony traumas; and (c) the improvement in the quality of life conferred by surgery. ${ }^{(36)}$ 
In young athletes with an acute anterior shoulder dislocation, immobilisation alone is not sufficient to allow adequate healing of the detached labrum. However, arthroscopic surgery followed by immobilisation can ensure a proper healing response. ${ }^{(4)}$ Some practitioners now believe that early surgical intervention in patients with first-time anterior shoulder dislocation is similar to the treatment of patients presenting with acute knee haemarthrosis. It is also widely accepted that diagnostic arthroscopy can be offered to young athletes with acute haemarthrosis, to assess the integrity of the anterior cruciate ligament and menisci. ${ }^{(4,37)}$ Furthermore, the conditions for surgical intervention are optimal after initial shoulder dislocation; the inflammatory reaction, together with its tissue factors, creates a healing environment that is ideal for soft tissue repair and there is also a reduced risk of capsular elongation, a condition usually seen in recurrent instability. ${ }^{(37)}$

\section{IS THERE A TREATMENT ALGORITHM?}

Currently, there is still no consensus in the literature with regard to the global management of patients presenting with first-time traumatic anterior dislocation of the shoulder. In Boone et al's literature review on the pathoanatomy of first-time anterior dislocation, early surgical repair was advocated in young patients less than 25 years old. ${ }^{(8)}$ In Speigl et al's study, ${ }_{\text {, }}^{(38)}$ a different treatment algorithm was applied for acute glenoid rim fractures caused by first-time traumatic anterior shoulder dislocations, i.e. surgery was performed if the patient had a bony Bankart lesion > 5\%, while conservative treatment was employed in all other cases. In this study, all 25 patients sustained a first-time traumatic anterior shoulder dislocation as a result of a ski or snowboard accident, and a bony Bankart lesion was detected in all shoulders. The primary outcome measure used was the Rowe score. Of the 25 patients, 12 underwent conservative treatment, while 13 underwent either an internal fixation procedure or a Latarjet procedure. The authors found that $83 \%$ of the patients who were treated conservatively had good to excellent outcomes, while $17 \%$ had moderate outcomes. For the patients who were treated surgically, $85 \%$ had good to excellent outcomes and 15\% had moderate outcomes. No recurrent dislocations were noted in both groups. ${ }^{(38)}$ While the results of Speigl et al's study are promising, it is limited by its retrospective nature and small sample size. There is thus a need for a large, randomised multicentre trial to validate the treatment algorithm for bony Bankart lesions proposed by Speigl et al.

\section{CONCLUSION}

The management of first-time traumatic anterior dislocation of the shoulder remains controversial. In general, conservative treatment of such dislocations has been shown to be associated with a high recurrence rate, especially in young athletes. Therefore, we believe that arthroscopic surgical stabilisation is a viable treatment option in young athletic patients with traumatic anterior shoulder dislocations. While there is some evidence that definitive surgical fixation of bony Bankart lesions of more than 5\% is associated with good outcomes, this treatment algorithm requires further validation through level I studies.

\section{REFERENCES}

1. Shah AS, Karadsheh MS, Sekiya JK. Failure of operative treatment for glenohumeral instability: etiology and causes. Arthroscopy 2011; 27:681-94.

2. Turkel SJ, Panio MW, Marshall JL, Girgis FG. Stabilizing mechanisms preventing anterior dislocation of the glenohumeral joint. J Bone Joint Surg Am 1981; 63:1208-17.

3. Rowe CR, Patel D, Southmayd WW. The Bankart procedure: a long-term end-result study. J Bone Joint Surg Am 1978; 60:1-16.

4. Wheeler JH, Ryan JB, Arciero RA, Molinari RN. Arthroscopic versus nonoperative treatment of acute shoulder dislocations in young athletes. Arthroscopy $1989 ;$ 5:213-7.

5. Pevny T, Hunter RE, Freeman JR. Primary traumatic anterior shoulder dislocation in patients 40 years of age and older. Arthroscopy 1998; 14:289-94.

6. Chahal J, Marks PH, Macdonald PB, et al. Anatomic Bankart repair compared with nonoperative treatment and/or arthroscopic lavage for first-time traumatic shoulder dislocation. Arthroscopy 2012; 28:565-75.

7. Robinson CM, Dobson RJ. Anterior instability of the shoulder after trauma. J Bone Joint Surg Br 2004; 86:469-79.

8. Boone JL, Arciero RA. First-time anterior shoulder dislocations: has the standard changed? Br J Sports Med 2010; 44:355-60.

9. Taylor DC, Arciero RA. Pathologic changes associated with shoulder dislocations. Arthroscopic and physical examination findings in first-time, traumatic anterior dislocations. Am J Sports Med 1997; 25:306-11.

10. Baker CL, Uribe JW, Whitman C. Arthroscopic evaluation of acute initial anterior shoulder dislocations. Am J Sports Med 1990; 18:25-8.

11. Antonio GE, Griffith JF, Yu AB, et al. First-time shoulder dislocation: High prevalence of labral injury and age-related differences revealed by MR arthrography. J Magn Reson Imaging 2007; 26:983-91.

12. Pollock RG, Wang VM, Bucchieri JS, et al. Effects of repetitive subfailure strains on the mechanical behavior of the inferior glenohumeral ligament. J Shoulder Elbow Surg 2000; 9:427-35.

13. Buscayret F, Edwards TB, Szabo I, et al. Glenohumeral arthrosis in anterior instability before and after surgical treatment: incidence and contributing factors. Am J Sports Med 2004; 32:1165-72.

14. Griffith JF, Antonio GE, Yung PS, et al. Prevalence, pattern, and spectrum of glenoid bone loss in anterior shoulder dislocation: CT analysis of 218 patients. AJR Am J Roentgenol 2008; 190:1247-54.

15. Wallace WA, Hellier M. Improving radiographs of the injured shoulder. Radiography 1983; 49:229-33.

16. Chandnani VP, Yeager TD, DeBerardino T, et al. Glenoid labral tears: prospective evaluation with MRI imaging, MR arthrography, and CT arthrography. AJR Am J Roentgenol 1993; 161:1229-35.

17. Kuhn JE. Treating the initial anterior shoulder dislocation--an evidencebased medicine approach. Sports Med Arthrosc 2006; 14:192-8.

18. Hovelius L, Augustini BG, Fredin H, et al. Primary anterior dislocation of the shoulder in young patients. A ten-year prospective study. J Bone Joint Surg Am 1996; 78:1677-84.

19. Simonet WT, Cofield RH. Prognosis in anterior shoulder dislocation. Am J Sports Med 1984; 12:19-24.

20. Kiviluoto O, Pasila M, Jaroma H, Sundholm A. Immobilization after primary dislocation of the shoulder. Acta Orthop Scand 1980; 51:915-9.

21. Seybold D, Schliemann B, Heyer CM, Muhr G, Gekle C. Which labral lesion can be best reduced with external rotation of the shoulder after a first-time traumatic anterior shoulder dislocation? Arch Orthop Trauma Surg 2009; 129:299-304.

22. Itoi $E$, Hatakeyama $Y$, Sato $T$, et al. Immobilization in external rotation after shoulder dislocation reduces the risk of recurrence. A randomized controlled trial. J Bone Joint Surg Am 2007; 89:2124-31.

23. Gumina S, Postacchini F. Anterior dislocation of the shoulder in elderly patients. J Bone Joint Surg Br 1997; 79:540-3.

24. Hovelius L, Olofsson A, Sandström B, et al. Nonoperative treatment of primary anterior shoulder dislocation in patients forty years of age and younger. a prospective twenty-five-year follow-up. J Bone Joint Surg Am 2008; 90:945-52.

25. Sachs RA, Lin D, Stone ML, Paxton E, Kuney M. Can the need for future surgery for acute traumatic anterior shoulder dislocation be predicted? J Bone Joint Surg Am 2007; 89:1665-74.

26. Wintzell G, Haglund-Akerlind Y, Ekelund A, et al. Arthroscopic lavage reduced the recurrence rate following primary anterior shoulder dislocation. A randomised multicentre study with 1-year follow-up. Knee Surg Sports TraumatolArthrosc 1999; 7:192-6.

27. Robinson CM, Jenkins PJ, White TO, Ker A, Will E. Primary arthroscopic 
stabilization for a first-time anterior dislocation of the shoulder A randomized, double-blind trial. J Bone Joint Surg Am 2008; 90:708-21.

28. Valentin A, Winge S, Engström B. Early arthroscopic treatment of primary traumatic anterior shoulder dislocation. A follow-up study. Scand J Med Sci Sports 1998; 8:405-10.

29. Law BK, Yung PS, Ho EP, Chang JJ, Chan KM. The surgical outcome of immediate arthroscopic Bankart repair for first time anterior shoulder dislocation in young active patients. Knee Surg Sports TraumatolArthrosc 2008; 16:188-93.

30. Bottoni CR, Wilckens JH, DeBerardino TM, et al. A prospective, randomized evaluation of arthroscopic stabilization versus nonoperative treatment in patients with acute, traumatic, first-time shoulder dislocations. Am J Sports Med 2002; 30:576-80.

31. Kirkley A, Werstine R, Ratjek A, Griffin S. Prospective randomized clinical trial comparing the effectiveness of immediate arthroscopic stabilization versus immobilization and rehabilitation in first traumatic anterior dislocations of the shoulder: long-term evaluation. Arthroscopy 2005; 21:55-63.

32. Jakobsen BW, Johannsen HV, Suder P, Søjbjerg JO. Primary repair versus conservative treatment of first-time traumatic anterior dislocation of the shoulder: a randomized study with 10-year follow-up. Arthroscopy 2007; 23:118-23.
33. Yanmis I, Tunay S, Kömürcü $M$, et al. Outcomes of acute arthroscopic repair and conservative treatment following first traumatic dislocation of the shoulder joint in young patients. Ann Acad Med Singapore 2003; 32:824-7.

34. Edmonds G, Kirkley A, Birmingham TB, Fowler PJ. The effect of early arthroscopic stabilization compared to nonsurgical treatment on proprioception after primary traumatic anterior dislocation of the shoulder. Knee Surg Sports TraumatolArthrosc 2003; 11:116-21.

35. Kirkley A, Griffin S, Richards C, Miniaci A, Mohtadi N. Prospective randomized clinical trial comparing the effectiveness of immediate arthroscopic stabilization versus immobilization and rehabilitation in first traumatic anterior dislocations of the shoulder. Arthroscopy 1999; 15:507-14.

36. Baumgarten KM, Wright RW. Ease of tying arthroscopic knots. J Shoulder Elbow Surg 2007; 16:438-42.

37. Barber FA, Ryu RK, Tauro JC. Should first time anterior shoulder dislocations be surgically stabilized? Arthroscopy 2003; 19:305-9.

38. Spiegl UJ, Ryf C, Hepp P, Rillmann P. Evaluation of a treatment algorithm for acute traumatic osseous Bankart lesions resulting from first time dislocation of the shoulder with a two year follow-up. BMC Musculoskelet Disord 2013; 14:305 


\section{SINGAPORE MEDICAL COUNCIL CATEGORY 3B CME PROGRAMME} (Code SMJ 201410A)

1. The glenohumeral joint is an inherently unstable joint because the small humeral head articulates with a deep glenoid fossa.

2. The glenohumeral joint is the most frequently dislocated joint in the human body.

3. The primary restraint to anterior humeral translation is the superior glenohumeral ligament.

4. A Bankart lesion is an avulsion of the labrum from the glenoid rim.

5. The static stabilisers of the glenohumeral joint include the glenoid fossa, labrum, joint capsule and glenohumeral ligaments.

6. The rotator cuff muscles and the triceps are dynamic stabilisers of the glenohumeral joint.

7. Anterior shoulder dislocations usually occur with the arm in an adducted and internally rotated position.

8. The Hill-Sachs lesion is a soft tissue lesion associated with a traumatic anterior shoulder dislocation.

9. The Bankart lesion is more common than the Hill-Sachs lesion in first-time anterior traumatic dislocations of the glenohumeral joint.

10. Repetitive loading of the inferior glenohumeral ligament induces laxity and reversible elongation in the ligament.

11. The study by Buscayret et al showed that the development of postoperative arthritis correlated with a higher number of preoperative dislocations.

12. The status of the axillary nerve should be clearly documented by the examining doctor during the clinical assessment of a patient with acute anterior dislocation of the glenohumeral joint, as well as after manipulation and reduction.

13. In an acute anterior glenohumeral dislocation, radiographs in the serendipity view should be requested to confirm the dislocation and presence of any associated fractures.

14. Post-reduction radiographs are not necessary as long as the glenohumeral joint appears clinically reduced and has a good range of motion that is painless.

15. The 'safe position' for the manipulated and reduced shoulder is in abduction.

16. Younger patients who are involved in contact or collision sports, or require overhead occupational use of the arm, are more likely to have recurrent dislocation of the shoulder, as compared to their less-active peers.

17. An unacceptable high risk of recurrence in the young athletic population is one of the major reasons cited in the literature for the support of immediate surgical stabilisation in acute traumatic anterior dislocations of the glenohumeral joint.

18. Conservative treatment has no role in first-time traumatic anterior dislocation of the glenohumeral joint.

19. Young athletic patients may benefit from early arthroscopic surgical stabilisation.

20. Definitive surgical fixation of bony Bankart lesions of more than $5 \%$ could be associated with good outcomes.

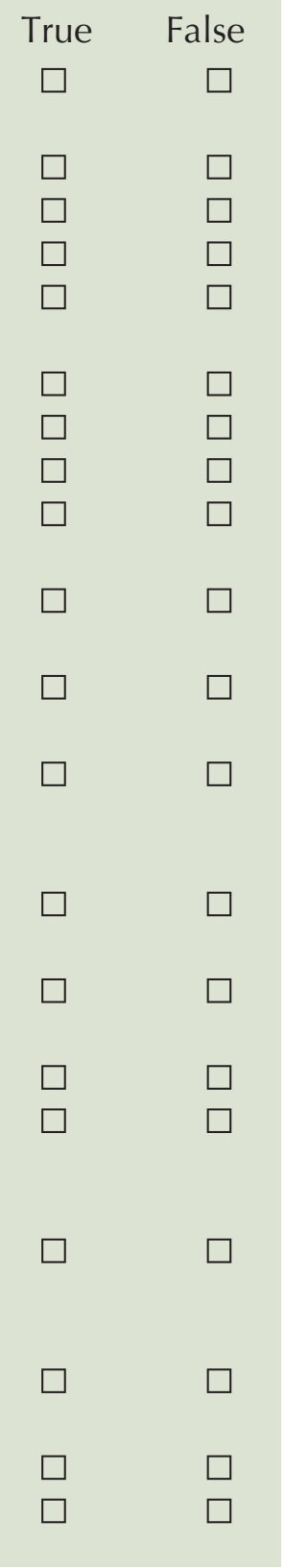

\footnotetext{
Doctor's particulars:

Name in full

MCR number

Email address

Specialty:

SUBMISSION INSTRUCTIONS:

(1) Log on at the SMJ website: http://www.sma.org.sg/publications/smjcurrentissue.aspx and select the appropriate set of questions. (2) Provide your name, email address and MCR number. (3) Select your answers and click "Submit".

RESULTS:

(1) Answers will be published in the SMJ December 2014 issue. (2) The MCR numbers of successful candidates will be posted online at the SMJ website by 9 December 2014. (3) Passing mark is $60 \%$. No mark will be deducted for incorrect answers. (4) The SMJ editorial office will submit the list of successful candidates to the Singapore Medical Council.

(5) One CME point is awarded for successful candidates.

Deadline for submission: (October 2014 SMJ 3B CME programme): 12 noon, 2 December 2014.
} 